\title{
The Frequency of Using Websites and Social Media by Various Age Groups to Form Opinions about Scientific Topics: Findings from the European Context
}

\author{
Izabela Warwas \\ University of Lodz \\ izabela.warwas@uni.lodz.pl
}

\author{
Małgorzata Dzimińska \\ University of Lodz \\ malgorzata.dziminska@uni.lodz.pl
}

\author{
Aneta Krzewińska \\ University of Lodz \\ aneta.krzewinska@uni.lodz.pl
}

\begin{abstract}
This article presents the frequency of using websites and social media by three age groups (18-34, 35-54, 55+) from Italy, Poland, Portugal, Slovakia, and Spain to form opinions about scientific topics. The findings come from studies performed within a European project entitled CONCISE, which is researching the role of communication in creating EU citizens' perceptions about science. The results show that young, heavy users take the lead in using all digital sources, except for general websites, where the middle-aged show dominance. Facebook is the most-used social media platform among all age groups. The group of social media non-users among older adults is almost seven times larger than that of the young. The study offers insights for scientists and policymakers which digital media to target to effectively reach the various age groups with reliable and trustworthy scientific information thus combating misinformation and pseudoscience.
\end{abstract}

Keywords: young, adults, elderly, social media, public consultation, science communication

\section{Introduction}

People's beliefs and perceptions have a great influence on the economy, politics, and culture [1]. However, evidence shows that people's opinions are at odds with the broad scientific consensus. The popularity of beliefs and perceptions that reject the scientific findings related to climate change, GMOs, complementary and alternative medicine (CAM), or vaccines poses threats to environmental situations and global health [2]-[5]. In order to understand how the public obtains scientific information and builds its attitudes, opinions, and beliefs, a Europe-wide research project, CONCISE, was created. A set of public consultations was designed to gather data directly from citizens. Public consultations were held with 497 citizens between September and
November 2019 in five EU countries: Italy, Poland, Portugal, Slovakia, and Spain. The topics chosen for discussion during the events included the most controversial issues, as reported in the literature: GMOs, climate change, vaccines, and CAM. The consultations aimed specifically at understanding the role of communication in forming EU citizens' perceptions of and beliefs about science.

Citizens' opinions are usually not directly derived from scientific information, as scientific messages usually go through a series of filters and mediators, affecting their final shape, and how, in the end, citizens view scientific topics. Websites and social media (SM) play an increasingly important role as key channels in informing the public and influencing their opinions. Globally, digital consumers now spend an average of 2 hours and 24 minutes per day on social networks and messaging apps [6, p. 4]. In 2020 the active global SM population worldwide amounted to 3.81 billion [7], and the average internet user has eight SM accounts, up from three in 2012 [6, p. 9]. Facebook is the most owned account, with YouTube in a strong second place. The number of daily active Instagram Stories users changed from $100 \mathrm{mln}$ to $500 \mathrm{mln}$ between October 2016 and January 2019 [8].

The current study fills the gap in the literature by presenting preferences on the digital media (DM) sources used by various age groups specifically to form opinions about scientific topics. The objectives of the study include: 1) defining which age groups are characterized by the highest number of heavy (frequent) website and SM users; 2) verifying if there is a relationship between the age of the user and the frequency of using websites and SM to form opinions about scientific topics, and finally 3) comparing the digital and social media indexes to understand how the members of the various age groups (18-34, 35-54, 55+) report website and social media use as sources and channels through which their perceptions, opinions, and beliefs about scientific topics are formed.

Answers to the posed research questions offer a new and useful input to develop science communication 
strategies to combat fake news and pseudoscience in a thoughtful and precise manner with the use of digital media. The findings can give clues to scientists, journalists, science communicators, NGOs, and policymakers about which websites and SM could be used as channels for communication campaigns on scientific findings targeted at different age groups.

\section{Terms and definitions}

Three age groups of citizens are analyzed in the study: young (18-34), middle-aged (35-54) and older adults (55+). The terms "elderly," "senior users," or "older adults" are used in the literature interchangeably [9, p. 130]. The division between young, middle-aged, and older adults is not that clear in the literature. For example, according to Wagner, Hassanein, and Head, the definitions of older adults vary considerably and denote seniors "over 40" on the lower end of the scale and "over 75" on the higher end of the scale [10]. Besides, there is a growing critique of treating the elderly as a homogeneous group without accounting for the diversity of their socio-economic status, motivations, and digital skills. The latest studies of seniors and their ICT (Information and Communication Technology) skills led to the development of categories of older adults to further differentiate their qualities [11]. Similar limitations need to be accounted for in the case of the other two groups. The age ranges proposed in our study are similar to the divisions adopted in other studies. For the young, Shensa et al. adopted the age range from 18 to 32 [12], and for seniors, some studies regarded them as older or equal to 55 years [13], [14]. Therefore, the definitions of the age groups that the authors of this study chose to apply (18-34, 35-54, 55+) follow the divisions found in the literature and will be used with an awareness of the limitations of such a generalization.

In contrast to the millennial generation, who are often referred to as "digital natives", the elderly are regarded as "digital immigrants" [15]. The literature points to the concept of age divide defined as a notion that distinguishes Internet users and non-users, on the one hand, and that differentiates the varying skill levels among ICT users, on the other [16]. In the context of older adults, a new term, the so-called grey divide, has been introduced to refer to the digital divide within the senior citizen sector itself. The division is visible in the access to and use of ICT [17]. The disparities are related to the socioeconomic status of the elderly, their level of education, or the family situation [18]. Namely, the higher the economic status and education level, and the stronger and wider the family ties with younger generations who can foster the development of ICT skills, then the higher the media literacy among the seniors. Past professional experience linked to using ICT skills and the availability of adequate training that is age-appropriate also play supportive roles for the elderly to become digitally literate. However, it is also important to note that studies on the digital divide have found that autonomous individuals that have unlimited Internet access and possess the required ICT skills do not receive the same benefits from Internet use [19]. This disparity can be determined by how people see their needs or desires, by their social and psychological determinants, and by the attributes of the media.

Digital media (DM) is an umbrella term for all types of electronic data (text, databases, images, audio, and video). Popular examples of DM include video or online games, software, videos, audio (e.g., streaming music or MP3s, audiobooks), ebooks, websites, and social media. The latest studies from 2020 show that $97 \%$ of digital consumers had used a social media network in the previous month. This means that being an Internet user means being a social media user [6, p. 7].

Social media is understood as "a collection of software-based digital technologies - usually presented as apps and websites - that provide users with digital environments in which they can send and receive digital content or information over some type of online social network" [20, p. 80]. Social media use is activities and practices among communities of people who gather online to share information, knowledge, and opinions using conversational media [21].

Social Networking Sites (SNS) are associated with the use of internet-based technologies to facilitate social interactions. "They enable members with common interests, activities, or real-life connections to form virtual online communities whereby they can intermix, mingle, interact and connect on a variety of levels" [22, p. 154]. Popular social networking sites include Facebook, Twitter, LinkedIn, Wikipedia, and YouTube. Social media allow individuals to create, distribute, and share information (Web 2.0), which facilitates a multi-way communication, in contrast to only searching for relevant information and passively looking at the content (Web 1.0) [23]. Social media can also be perceived as places where people conduct significant parts of their lives, which means that SM becomes less about the specific technologies or platforms, and more about what people do in these environments. "Social media has essentially become almost anything - content, information, behaviors, people, organizations, institutions - that can exist in an interconnected, networked digital environment where interactivity is possible" [20, p. 80]. In this study, the authors will refer to social media as various Web 2.0 applications that allow for the creation and sharing of 
content. The use of SM will be analyzed in the context of forming opinions about scientific topics. In this study, forming opinions is understood as shaping beliefs, attitudes, and perceptions, and it follows the definition by Fazio [24], who identifies three key components for the development of an attitude: (1) affection, (2) cognition, and (3) behavioral intention [25]. The affective and cognitive components of an attitude concern feelings, beliefs, and, ultimately, the evaluation of a situation, concept, or object, whereas the behavioral component refers to an intended action.

\section{Frequency of using social media by young, middle-aged and older adults}

The study involves an analysis of the frequency of using the following social media: YouTube, Twitter, Facebook, Instagram, and blogs. The choice of SNSs was based on the popularity of these sites to communicate scientific findings. What follows in this section is the contextual information on the nature of SM use by various age groups.

Social media users usually maintain a presence across several platforms. While the average Internet user had about 6.2 social media accounts in 2015, the figure has risen to nearly 8 in 2020 [6, p. 9]. This multinetworking is a response to the widening choice of platforms, but it is also being caused by the degree of specialization, where some users are turning to particular platforms to carry out certain types of networking behaviors. In 2020, Facebook (FB) (est. in 2004) had the most active monthly users (2.5 billion). FB helps people connect with friends and family, maintain long-distance relationships, and it also allows private messaging, public commenting, personal broadcasting, and photo-sharing. YouTube was launched in 2005 ( 2 billion users in 2020), and it is used mainly for multimedia sharing. Instagram users focus on uploading pictures and posting statuses. This social network was established in 2010, and by 2020, it had reached 1 billion users. Twitter (launched in 2006) allows people to upload pictures and post statuses. It also enables brief personal broadcasts, and it had reached 386 million monthly active users in 2020 [26]. Meanwhile, leading science blogs can attract up to 1.5 million visitors and several thousand comments every month [27]. These statistics show the growth of SM use, and at the same time, they highlight their importance as a source and a channel of scientific communication.

Media use is connected with demographic factors, including age [28]. The analysis of SM use by generation in Europe shows an increase in all age groups in terms of average time spent on SM per day in hours and minutes (hh:mm), with the elderly taking the lead as the group that advanced most in this respect (Table 1).

Table 1. Average time spent on SM by age groups in 2019 in Europe

\begin{tabular}{|l|c|r|r|}
\hline \multirow{2}{*}{ Age (Generation) } & \multicolumn{2}{|c|}{$\begin{array}{c}\text { Average time spent on } \\
\text { social media }\end{array}$} & \multirow{2}{*}{$\begin{array}{c}\text { increase } \\
2015-2019\end{array}$} \\
\cline { 2 - 3 } & 2015 & 2019 & $+16 \%$ \\
\hline $16-22(\mathrm{Z})$ & $2: 41$ & $3: 07$ & $+6 \%$ \\
\hline $23-36$ (Millenials) & $2: 19$ & $2: 28$ & $+13 \%$ \\
\hline $37-55(\mathrm{X})$ & $1: 19$ & $1: 29$ & $+20 \%$ \\
\hline $56-64$ (Baby boomers) & $0: 51$ & $1: 01$ & \\
\hline
\end{tabular}

Source: Social media by generation [29]

Social media users of different ages also vary in terms of the number of SM platforms they network on. Table 2 presents the data divided into age groups, proving that all age groups increased their multinetworking habits and that the older adults changed their average number of SNSs most prominently in comparison to the other age groups.

Table 2: Multi-networking by age groups globally

\begin{tabular}{|c|c|c|c|}
\hline \multirow[t]{2}{*}{ Age (Generation) } & \multicolumn{2}{|c|}{$\begin{array}{l}\text { Average number of } \\
\text { SM accounts }\end{array}$} & \multirow{2}{*}{$\begin{array}{l}\% \text { increase } \\
2015-2019\end{array}$} \\
\hline & 2015 & 2019 & \\
\hline $16-22(Z)$ & 5.9 & 9.4 & $+37 \%$ \\
\hline 23-36 (Millenials) & 6.3 & 9.8 & $+36 \%$ \\
\hline $37-55(\mathrm{X})$ & 4.9 & 7.6 & $+36 \%$ \\
\hline 56-64 (Baby boomers) & 3.0 & 5.3 & $+43 \%$ \\
\hline
\end{tabular}

Source: Social media by generation [29]

The above-presented review reveals the frequency of using SM by different age groups. However, what turned out to be missing in the literature and is addressed in this study concerns the analysis of the frequency in the context of forming opinions about scientific topics. This is especially important for the field of science communication because a good recognition of the DM preferences by age groups can result in more accurate outreach to the chosen target audience and thus more effective actions aiming at combating pseudoscience.

\section{Using social media to form opinions about scientific topics}

Empirical studies revealed that messages on SM can influence people's post-exposure opinions, attitudes, and beliefs on the corresponding topics [30]-[34]. Media communication theories and approaches [35] help understand and interpret the blending of mass and personal communication that social platforms offer. Five attributes of contemporary electronic opinion environments can change the way users form and express opinions: the juxtaposition of mass media and user-generated content, the ideological homogeneity and heterogeneity of online networks, the technical ease 
with which the users express opinions, the reach of messages, and the networked audiences [36]. The latest studies further explain, analyze, and model the dynamics of the change of opinions on social networks [37], [38]. Meanwhile, the findings of cognitive science contribute by informing the design of information architectures that encourage the dissemination of highquality information that discourages the spread of misinformation [4].

The findings from studies on using SM to form opinions about scientific topics exemplify the attributes of SM users. The studies highlight that the perceived social consensus, which is visible through the number of followers, comments, likes, or views, supports opinion formation in favor of the one that is shared by the majority. It was confirmed in the studies by Lewandowsky et al. [39], who examined how blog posts and comments interact to affect readers' attitudes and beliefs concerning the scientifically well-established fact that greenhouse gas emissions are warming the Earth. The users' perception of how widely an opinion expressed in a blog post is endorsed and shared by other readers proved most powerful in shaping and changing people's attitudes and beliefs. Similarly, the number of viewers of a YouTube video can be interpreted as a sign of the issue's importance in the public eye [40]. The tendencies of following the majority can be interpreted with the concept of conformity, defined as the act of matching one's behavior to the responses of others [41]. Conformity can be driven by informational motivations, i.e., the desire to interpret reality in an accurate way or normative motivations that are based on the desire to obtain social approval from others [42].

The growing amount of purposefully spread misinformation and pseudoscience on SM is recognized as an increasingly worrying issue [3], [4], [43]. The practices of influencing SM metrics by manipulation or purchasing to expand the outreach are widespread [44]. The number of followers can be inflated by various means, i.e., buying tweets from influencers or using SM manipulation software.

Zielinska [45] reports that in case of GMOrelated concerns, what differentiates the behavior of the two groups of pro- and anti-GMO activists on SM is the frequency of posting messages, the tone of their narratives, or the volume of members grouped under each label. Rational, scientific content is presented only when new facts arrive, or when legislation changes, while emotional FB posts of anti-GMO supporters are frequent and focus on building traffic and a high volume of followers. In contrast to pro-GMO activists, the antiGMO activists are better organized, cite each other often, and recruit single-minded scientists, celebrities, or politicians to gain popularity of their views [45, p. 4]. Kata [43] reports a series of tactics that are employed by anti-vaccination activists to gain an audience and spread their message. They skew scientific findings, shift hypotheses, censor facts, or attack the opposition. Specifically, they reject science that fails to support antivaccine positions and endorse poorly-conducted studies that promote anti-vaccine agendas. They propose new theories for vaccines, suppressing dissenting opinions, or shutting down critics by attacking them via both personal insults or by filing legal actions [43, p. 3781].

The readers/viewers' lack of scientific background can make it difficult for them to distinguish between trustworthy and untrustworthy sources, real news and fake news [46]-[50]. Thus, pseudoscience becomes widespread, and misinformation on SM is a cause of growing concern [51].

\section{Methodology}

This research study is guided by the following research questions:

RQ1: Which age group(s) are characterized by the highest number of heavy (frequent) users of websites and social media?

$R Q 2:$ Is there a relationship between the age of the users and the frequency of using websites and social media to form opinions about scientific topics?

RQ3: What are the digital and social media indexes for three age groups (18-34, 35-54, 55+)?

Citizen public consultations provided data from almost five hundred participants to find answers to the posed questions. The methodological inspiration for conducting the consultative meetings under the CONCISE project was the World Wide Views (WWVs) method, created by the Danish Technology Council and first used in 2009 during a debate on global warming. In its original form, meetings organized with the use of WWVs focused on transnational issues (biodiversity, climate, energy), but the same method can also be applied at a national or even local level [52].

As part of the CONCISE project, which provided the empirical basis for the preparation of this article, consultation meetings were held in five European countries in which 497 citizens participated circa 100 people from each country. The selection process of citizens for the CONCISE consultations in each of the five countries was as inclusive as possible to represent the diversity of the societies. The factors that were taken into account were: gender, age (divided into six groups: 18-24, 25-34, 35-44, 45-54, 55-64, 65+), level of studies (no education, primary education, secondary, higher), place of residence (urban and rural areas), special needs, national minorities (determined for each country separately depending on which minorities live in the country), and immigrant population. In the recruitment process, the proportions 
between different categories of participants in terms of gender, education, place of residence, and age were monitored to be as close as possible to the ideal sample that assumed national typological representativeness. However, due to various recruitment challenges (motivational, organizational and logistical), in the end, the final sample deviated from the assumed one, but remained inclusive and reflected the core diversities of the societies.

The participants were assigned to tables in such a way that there were 8 to 10 people at each table who were under the care of a facilitator (leading the discussion) and an observer who noted the order in which they took the floor (which was important for transcription). They discussed a given topic for an hour and then answered questions provided in the form of a short questionnaire on the topics discussed. This procedure was repeated four times to cover each topic (CAM, GMO, vaccines, and climate change), and the individual rounds were separated by breaks. Thus, the WWV method was adapted to the needs of the project and the organizational capabilities of the organizers.

Data presenting answers to the questionnaire items that were related to the frequency of using websites and SM to form opinions about scientific topics were collected, coded, anonymized, and prepared for quantitative analysis. Statistical analyses were then carried out with the use of SPSS. The analyses included: analysis of crosstabs with the use of the $\mathrm{Chi}^{2}$ test and Cramer's V coefficient and one-way analysis of variance (ANOVA).

\section{Results}

The consultation participants completed two short questionnaires after two rounds of discussion. They answered questions about the sources of information on the four topics discussed, i.e., climate change, vaccines, CAM, and GMOs. In this way, 936 answers from 497 participants of the consultations in 5 European countries were collected, spread almost equally in individual countries (Italy 19,2\%, Poland $21,4 \%$, Portugal $21,7 \%$, Slovakia 16,2\%, Spain $21,5 \%$, out of all the answers).

Nearly $60 \%$ of the group were women, while men constituted $41 \%$. Most of the respondents, were well-educated, as $55.6 \%$ had higher education, $38.7 \%$ had secondary education, and the remainder $(5.7 \%)$ had completed primary education. The attendees were almost three times more likely to be urban residents (72.8\%) than inhabitants of rural areas (27.2\%). For the analysis, the respondents were divided into three age groups: young, from 18 up to 34 years old (32.2\%), middle-aged, between 35 and 54 years old (38.0\%), and older adults, 55 and older $(29.8 \%)$.

In the first part of the study, the answer to the following research question was sought: Which age group(s) are characterized by the highest number of heavy (frequent) digital and social media users? The participants declared how often they look for information on scientific topics using the following online resources: general websites, information websites, social media, i.e., Facebook, YouTube, Twitter, and blogs, which were chosen based on their popularity [7], and their appropriateness to present scientific findings, i.e., the websites or blogs. In assessing each of these sources, the consultation participants used a scale from "every day" to "never." The results in the "every day" section were selected for analysis because they denoted the heavy users, i.e., people who very often search for scientific content online.

Table 3: The percentage of everyday DM users by age groups

\begin{tabular}{|l|r|r|r|}
\hline \multirow{2}{*}{ Digital media (DM) } & \multicolumn{3}{|c|}{ Age groups } \\
\cline { 2 - 4 } & $18-34$ & $35-54$ & \multicolumn{1}{|c|}{$55+$} \\
\hline General websites & 20.1 & 20.3 & 10.4 \\
\hline Information websites & 19.1 & 16.7 & 10.0 \\
\hline YouTube & 16.0 & 5.8 & 4.4 \\
\hline Twitter & 8.9 & 6.8 & 8.4 \\
\hline Facebook & 24.9 & 17.2 & 13.1 \\
\hline Instagram & 20.7 & 6.8 & 5.8 \\
\hline Blogs & 7.7 & 5.5 & 6.0 \\
\hline
\end{tabular}

The overall conclusion is that the young heavy users take advantage of all the examined options to form their opinion on scientific topics more frequently than the older heavy users. The big difference between the young and the older adult users primarily concerns YouTube and Instagram, where the young, everyday users of these sites are more than three times more active than the older adults. It is also worth noting that the middle-aged are more similar to the young in their online behavior when general and information pages are considered, while they resemble the older adults more when it comes to using SM like Instagram and blogs. It is also worth noting that there is little difference in the everyday use of Twitter between the different age groups. It turns out that $8.9 \%$ of the young, $6.8 \%$ of the middle-aged, and $8.4 \%$ of the older adults use this app on an everyday basis.

Research question 2 asks: What is the relationship between the age of the citizens and the frequency of their use of digital and social media to form opinions about scientific topics? Statistical analyses of whether age affects the frequency of using specific channels offered by DM were carried out. In six out of seven channels (except for Twitter), these relationships 
are statistically significant with a weak or medium dependency strength. The appropriate $\mathrm{Chi}^{2}$ test measures and significance levels are summarized in Table 4.

Table 4: $\mathrm{Chi}^{2}$ test measures, their significance levels, and Cramer's Values for each channel

\begin{tabular}{|l|r|r|r|}
\hline \multicolumn{1}{|c|}{ Channels } & $\begin{array}{c}\text { Chi-Square } \\
\text { tests value }\end{array}$ & \multicolumn{1}{c|}{ Significance } & $\begin{array}{c}\text { Cramer's V } \\
\text { value }\end{array}$ \\
\hline General website & 38.68 & 0.001 & 0.15 \\
\hline Information website & 74.37 & 0.001 & 0.21 \\
\hline YouTube & 64.48 & 0.001 & 0.19 \\
\hline Twitter & 8.70 & 0.31 & - \\
\hline Facebook & 83.30 & 0.001 & 0.22 \\
\hline Instagram & 100.61 & 0.001 & 0.25 \\
\hline Blogs & 13.91 & 0.05 & 0.09 \\
\hline
\end{tabular}

Research question 3 guided the development of digital media and social media indexes: What are the digital and social media indexes for three age groups (18-34, 35-54, 55+)? Based on the question about the frequency of using various websites and SNSs, an index was created that represents the extent of engagement in using digital media to form opinions about scientific topics. The following procedure was adopted: one point was awarded to every respondent who has ever visited a website or SNS and 0 points for those who did not show such an activity. Then the points were added up in two ways. In the first - referring to digital media - visiting websites and SNSs were taken into account, i.e., general websites, information websites, YouTube, Twitter, Facebook, Instagram, and blogs. This index ranged from 0 (for respondents who did not use any of these online news media) to 7 (for those who used all of the media channels) and was named Digital Media Index (DMI). In the case of the second index (SMI - Social Media Index), only social media were included (YouTube, Twitter, Facebook, Instagram, and blogs), and values from 0 (for non-users) to 5 (for heavy users) were assigned. Then, individual index values were assigned to the three age groups of the respondents - the young, middle-aged, and older adults. Detailed results are presented in Table 5 and Table 6.

Table 5: The percentage of consultation participants by age groups and their DMI

\begin{tabular}{|c|r|r|r|}
\hline \multirow{2}{*}{$\begin{array}{c}\text { Digital Media Index } \\
\text { (DMI) }\end{array}$} & \multicolumn{3}{|c|}{ Age groups } \\
\cline { 2 - 4 } & $18-34$ & \multicolumn{1}{c|}{$35-54$} & \multicolumn{1}{c|}{$55+$} \\
\hline 0 (non-user) & 2.3 & 3.1 & 20.8 \\
\hline 1 & 2.7 & 5.9 & 13.3 \\
\hline 2 & 4.7 & 12.1 & 16.1 \\
\hline 3 & 11.3 & 17.7 & 14.0 \\
\hline 4 & 15.0 & 20.8 & 7.5 \\
\hline 5 & 21.6 & 19.4 & 12.2 \\
\hline 6 & 19.9 & 8.1 & 6.8 \\
\hline 7 (heavy user) & 22.6 & 12.9 & 9.3 \\
\hline
\end{tabular}

Table 6: The percentage of consultation participants by age groups and their SMI

\begin{tabular}{|c|r|r|r|}
\hline \multirow{2}{*}{$\begin{array}{c}\text { Social Media Index } \\
(\text { SMI) }\end{array}$} & \multicolumn{3}{|c|}{ Age groups } \\
\cline { 2 - 4 } & $18-34$ & \multicolumn{1}{|c|}{$35-54$} & \multicolumn{1}{c|}{$55+$} \\
\hline 0 (non-user) & 6.0 & 14.6 & 41.2 \\
\hline 1 & 10.6 & 20.8 & 19.4 \\
\hline 2 & 16.9 & 21.9 & 9.7 \\
\hline 3 & 22.9 & 21.1 & 12.5 \\
\hline 4 & 20.3 & 8.1 & 7.2 \\
\hline 5 (heavy user) & 23.3 & 13.5 & 10.0 \\
\hline
\end{tabular}

It can be seen that the values of both indicators decrease with age - for the young, they adopt higher values, and for the older adults, lower values. This means that the young use a larger number of websites and SNSs to form opinions about scientific topics.

It is interesting to look at the two extremes of these indexes, i.e., the number of non-users and heavy users in particular age categories. Concerning DMI, the group of non-users among the older adults is ten times bigger than among the young adults. The middle-aged are similar in this respect to the young. In the case of social media, these differences are slightly smaller, and the group of non-users among the older adults is almost seven times larger than that of the young. When analyzing the size of individual age groups among heavy users, it turns out that there are two times more heavy users in the young group than in the group of older adults, but interestingly, in this case, the size of the group of middle-aged heavy users is closer to the number of social media users representing the older adults.

The study also involved checking if the differences in the number of channels used by the young, middle-aged and older adults are statistically significant, i.e., whether the average number of websites and social media to which the older adults have access significantly differs from the average number used by the young and the middle-aged. The analyses included calculations of the average values for individual indexes (Tables 5 and 6) and then the analysis of variance (ANOVA).

Table 7: The Mean of the DMI and SMI for the three age groups

\begin{tabular}{|l|r|r|r|}
\hline & $\begin{array}{c}\text { The } \\
\text { young }\end{array}$ & $\begin{array}{c}\text { The } \\
\text { middle- } \\
\text { aged }\end{array}$ & $\begin{array}{c}\text { The older } \\
\text { adults }\end{array}$ \\
\hline Digital Media Index & 4.91 & 4.03 & 2.85 \\
\hline Social Media Index & 3.11 & 2.28 & 1.55 \\
\hline
\end{tabular}

While young digital media users get information from an average of 4.91 sources and visit 3.11 social media platforms, the elderly look for information on 2.85 websites and network on 1.55 social 
media platforms. The ANOVA test value (for DMI $F_{(2,935)}=80.75, p<0.001$ and for SMI $F_{(2,935)}=68.81$, $\mathrm{p}<0.001)$ indicates that the differences in means between at least two groups are statistically significant for both indexes, and the post hoc tests showed that significant statistical differences occur in both indexes for each pair of groups (i.e. when we compare the young and the middle-aged, the young and the older adults, and the middle-aged with the older adults). Based on these analyses, it can be concluded that the age of the media users influences how frequently they use the internet to search for scientific content.

\section{Conclusion and discussion}

Our study focused on measuring how often various age groups use websites and SM as sources and channels through which the perceptions, opinions, and beliefs about scientific topics are formed. This study aimed to discover the key differences in the frequency of using digital sources by three age groups (18-34, 35-54, 55+).

In response to $R Q 1$ : Which age group $(s)$ are characterized by the highest number of heavy (frequent) website and social media users?, the results showed that the young heavy users take the lead in using all digital sources, except for general websites, where the middleaged show dominance. The older heavy users are the least frequent in all listed digital media. Young heavy users look for scientific information on Facebook, Instagram, general websites, and information websites, respectively. The middle-aged heavy users prefer to look for scientific news mainly on general websites and information websites, and FB. In the case of the older adults, the reverse situation is visible: their first choice is $\mathrm{FB}$, while the general and information websites follow. The interesting findings also include the fact that Facebook is the most often used SM platform among all age groups. What is more, there is little difference in the everyday use of Twitter between the different age groups. YouTube and Instagram are used three times more frequently by the young than by the older adults to form opinions about scientific topics.

The analyses related to the RQ2: Is there a relationship between the age of the users and the frequency of using websites and social media to form opinions about scientific topics? proved that age affects the frequency of media use. The analyses showed that in six out of seven channels (except for Twitter), these relationships are statistically significant with weak or medium dependency strength.

The findings related to $R Q 3:$ What are the digital and social media indexes for three age groups (18-34, 35-54, 55+)? also revealed valuable facts. The DMI shows that the group of non-users among the older adults is ten times bigger than among the young, while in the case of SMI, these differences are slightly smaller, and the group of non-users among older adults is almost seven times larger than that of the young.

The study confirms the relationship between the age and frequency of using websites and SM platforms. This research contributes to the existing literature by providing specific information that adds to the results obtained by other researchers. Specifically, it adds to the body of knowledge by presenting preferences on the DM sources used by various age groups to form opinions about scientific topics. This forms a useful signpost for scientists and journalists who propagate scientific findings, or for policymakers on where to get actively involved to provide arguments and effectively reach the target audience with reliable and trustworthy information.

The recommendations related to which digital sources to use with each of the three groups can be summarized as follows. Concerning the youngest group of recipients of scientific content (18-34 years old), it is recommended to use all digital media, as they take the lead in each medium. Scientific messages directed to the group of middle-aged users should be posted mainly on general websites and information websites, and also on FB. This group, however, is very diverse, so the choice of language of the communication and a wide thematic range will be decisive here. Considering the preferences of the older adults, publishers of scientific articles should direct them to FB as well as to websites, blogs, and Twitter. Special attention should be paid to Facebook, as it remains the most-used channel and source of scientific content among all age groups. Scientific content providers should, therefore, pay particular attention to using this platform and make it a priority in their portfolio of channels on which to share news.

The analyzed specifics of using DM allow us to formulate a recommendation related to using a multichannel approach to reach the target audiences. The field of science communication can capitalize on the growing number of digital users across all age groups. By tailoring the message format to the specificity of the medium and the preferences of the age groups for particular media, the communication process can become more accurate in terms of reaching the expected target audience. Scientific news providers also need to note that SM users pay attention to the quantitative evidence of the popularity of a given message, such as likes, tweets, the number of followers, or the number of comments [53]. With all this in mind, understanding the frequency of using websites and SM platforms constitutes an important element of a strategy to combat fake news and pseudoscience in a thoughtful and precise manner. 
The findings show the current use of websites and SM to form opinions about scientific findings. However, it is crucial to monitor the trends in this respect due to the changing nature of users' behaviors and the rapid development of digital media resources. The findings form the basis for further analysis and monitoring of trends in the use of social media platforms and websites by various age groups to form opinions about scientific findings.

\section{Acknowledgements}

The work presented in this paper was carried out as part of the project CONCISE: Communication role on perception and beliefs of EU Citizens about Science. This project has received funding from the European Union's Horizon 2020 research and innovation programme under grant agreement No 824537. The authors thank all the members of the CONCISE project consortium and highly appreciate the contribution from the teams led by Carolina Moreno (project coordinator from University of Valencia), Ana Delicado (University of Lisbon), Giuseppe Pellegrini (Observa), and L'ubomír Šottník (University of Trnava), who organized the consultations.

\section{References}

[1] Q. He, Z. Lei, X. Wang, M. Huang, and Y. Cai, "An effective scheme to address influence maximization for opinion formation in social networks," Trans. Emerg. Telecommun. Technol., vol. 30, no. 6, pp. 1-15, 2019.

[2] J. R. Kerr and M. S. Wilson, "Changes in perceived scientific consensus shift beliefs about climate change and gm food safety," PLoS One, vol. 13, no. 7, pp. 1-18, 2018.

[3] A. Kata, "A postmodern Pandora's box: Antivaccination misinformation on the Internet," Vaccine, vol. 28, no. 7, pp. 1709-1716, 2010.

[4] S. Lewandowsky, U. K. H. Ecker, and J. Cook, "Beyond Misinformation: Understanding and Coping with the 'Post-Truth' Era," J. Appl. Res. Mem. Cogn., vol. 6, no. 4, pp. 353-369, 2017.

[5] G. P. Chrousos and A.-F. A. Mentis, "Medical misinformation in mass and social media: an urgent call for action, especially during epidemics," Eur. J. Clin. Invest., no. February, p. e13227, 2020.

[6] Global Web Index, “Global Web Index’s flagship report on the latest trends in social media," 2020.

[7] Statista, "Social media - Statistics and Facts," 2020. [Online]. Available: https://www.statista.com/topics/1164/socialnetworks. [Accessed: 07-Jul-2020].

[8] Statista, "Number of daily active Instagram Stories users from October 2016 to January 2019," 2020.

[9] C. M. Q. Ramos, M. M. Mendonça, and J. M.
F. Rodrigues, "Senior users of social networks: Technological experience and use habits analysis," ACM Int. Conf. Proceeding Ser., pp. 129-136, 2018.

[10] N. Wagner, K. Hassanein, and M. Head, "Computer use by older adults: A multidisciplinary review," Comput. Human Behav., vol. 26, no. 5, pp. 870-882, 2010.

[11] A. Quan-Haase, C. Williams, M. Kicevski, I. Elueze, and B. Wellman, "Dividing the Grey Divide: Deconstructing Myths About Older Adults' Online Activities, Skills, and Attitudes," Am. Behav. Sci., vol. 62, no. 9, pp. 1207-1228, Aug. 2018.

[12] A. Shensa, C. G. Escobar-Viera, J. E. Sidani, N. D. Bowman, M. P. Marshal, and B. A. Primack, "Problematic social media use and depressive symptoms among U.S. young adults: A nationally-representative study," Soc. Sci. Med., vol. 182, pp. 150-157, 2017.

[13] T. J. Sinclair and R. Grieve, "Facebook as a source of social connectedness in older adults," Comput. Human Behav., vol. 66, pp. 363-369, 2017.

[14] T. Nef, R. L. Ganea, R. M. Müri, and U. P. Mosimann, "Social networking sites and older users - A systematic review," International Psychogeriatrics, vol. 25, no. 7. Cambridge University Press, pp. 1041-1053, Jul-2013.

[15] M. Milovich Jr. and D. Burleson, "Social Media and Older Adults: Understanding Cognitive Training and Social Network," Proc. 51st Hawaii Int. Conf. Syst. Sci., pp. 32823291, 2018.

[16] G. Nimrod, "Older audiences in the digital media environment," Inf. Commun. Soc., vol. 20, no. 2, pp. 233-249, Feb. 2017.

[17] T. N. Friemel, "The digital divide has grown old: Determinants of a digital divide among seniors," New Media Soc., vol. 18, no. 2, pp. 313-331, Feb. 2016.

[18] F. Mubarak and M. Nycyk, "Teaching older people internet skills to minimize grey digital divides: Developed and developing countries in focus," J. Information, Commun. Ethics Soc., vol. 15, no. 2, pp. 165-178, 2017.

[19] A. J. A. M. van Deursen and E. J. Helsper, "The Third-Level Digital Divide: Who Benefits Most from Being Online?," vol. 10, pp. 29-52, 2015.

[20] G. Appel, L. Grewal, R. Hadi, and A. T. Stephen, "The future of social media in marketing," J. Acad. Mark. Sci., vol. 48, no. 1, pp. 79-95, 2020.

[21] Safko L., The social media bible: tactics, tools 
\& strategies for business success, 2nd ed., vol. 50, no. 08. John Wiley \& Sons, 2013.

[22] F. Kamoun, S. Gharbi, and A. A. Ghazeli, "Reconnecting with the past: a framework to better serve the information needs of older people on social networking sites," Work. with Older People, vol. 22, no. 3, pp. 154-165, Sep. 2018.

[23] V. Parida, R. Mostaghel, and P. Oghazi, "Factors for Elderly Use of Social Media for Health-Related Activities," Psychol. Mark., vol. 33, no. 12, pp. 1134-1141, Dec. 2016.

[24] R. H. Fazio, "How do attitudes guide behavior," in Handbook of Motivation and Cognition, R. M. Sorrentino and E. T. Higgins, Eds. New York: Guilford, 1986, pp. 204-43.

[25] T. Daugherty, M. S. Eastin, and L. Bright, "Exploring Consumer Motivations for Creating User-Generated Content," J. Interact. Advert., vol. 8, no. 2, pp. 16-25, 2008.

[26] Statista, "Global social networks ranked by number of users," 2020. [Online]. Available: https://www.statista.com/statistics/272014/glob al-social-networks-ranked-by-number-ofusers/. [Accessed: 06-Jun-2020].

[27] S. A. Batts, N. J. Anthis, and T. C. Smith, "Advancing science through conversations: Bridging the gap between blogs and the academy," PLoS Biol., vol. 6, no. 9, pp. 18371841, 2008.

[28] H. Scherer, "Media Use by Social Variable," in The International Encyclopedia of Communication, John Wiley \& Sons, Ltd, 2012.

[29] Global Web Index, "Social media by generation," p. 2019, 2019.

[30] S. Winter, C. Brückner, and N. C. Krämer, "They Came, They Liked, They Commented: Social Influence on Facebook News Channels," Cyberpsychology, Behav. Soc. Netw., vol. 18, no. 8, pp. 431-436, Aug. 2015.

[31] A. A. Anderson, D. Brossard, D. A. Scheufele, M. A. Xenos, and P. Ladwig, "The "nasty effect:' online incivility and risk perceptions of emerging technologies," J. Comput. Commun., vol. 19, no. 3, pp. 373-387, 2014.

[32] J. B. Walther and J. woo Jang, "Communication Processes in Participatory Websites," J. Comput. Commun., vol. 18, no. 1, pp. 2-15, 2012.

[33] C. D. Stavrositu and J. Kim, “All Blogs Are Not Created Equal: The Role of Narrative Formats and User-Generated Comments in Health Prevention," Health Commun., vol. 30, no. 5, pp. 485-495, 2015.
[34] E. J. Lee, “That's Not the Way It Is: How User-Generated Comments on the News Affect Perceived Media Bias," J. Comput. Commun., vol. 18, no. 1, pp. 32-45, 2012.

[35] R. S. Fortner and P. M. Fackler, Eds., The Handbook of Media and Mass Communication Theory. Hoboken, NJ, USA: John Wiley \& Sons, Inc., 2014.

[36] G. Neubaum and N. C. Krämer, "Opinion Climates in Social Media: Blending Mass and Interpersonal Communication," Hum. Commun. Res., vol. 43, no. 4, pp. 464-476, 2017.

[37] A. Taalaibekova, "Opinion formation in social networks," Oper. Res. Decis., vol. 28, no. 2, pp. 85-108, 2018.

[38] B. Buechel, T. Hellmann, and S. Klößner, "Opinion dynamics and wisdom under conformity," J. Econ. Dyn. Control, vol. 52, pp. 240-257, Mar. 2015.

[39] S. Lewandowsky, J. Cook, N. Fay, and G. E. Gignac, "Science by social media: Attitudes towards climate change are mediated by perceived social consensus," Mem. Cogn., vol. 47, no. 8, pp. 1445-1456, 2019.

[40] J. T. Spartz, L. Y. F. Su, R. Griffin, D. Brossard, and S. Dunwoody, "YouTube, Social Norms and Perceived Salience of Climate Change in the American Mind," Environ. Commun., vol. 11, no. 1, pp. 1-16, 2017.

[41] R. B. Cialdini and N. J. Goldstein, "Social Influence: Compliance and Conformity," Annu. Rev. Psychol., vol. 55, no. 1, pp. 591-621, 2004.

[42] J. Colliander, "'This is fake news': Investigating the role of conformity to other users' views when commenting on and spreading disinformation in social media," Comput. Human Behav., vol. 97, no. March, pp. 202-215, 2019.

[43] A. Kata, "Anti-vaccine activists, Web 2.0, and the postmodern paradigm - An overview of tactics and tropes used online by the antivaccination movement," Vaccine, vol. 30, no. 25, pp. 3778-3789, 2012.

[44] NATO, "The Black Market for Social Media Manipulation," NATO Strateg. Commun. Cent. Excell., p. 20, 2018.

[45] I. Zielińska, "Wpływ mediów społecznościowych na komunikację naukową: strategie przeciwników GMO na Facebooku," Adeptus, no. 10, 2017.

[46] M. Baker, "1,500 scientists lift the lid on reproducibility," Nature, vol. 533, no. 7604, pp. 452-454, May 2016. 
[47] A. Ceron, L. Curini, S. M. Iacus, and G. Porro, "Every tweet counts? How sentiment analysis of social media can improve our knowledge of citizens' political preferences with an application to Italy and France," New Media Soc., vol. 16, no. 2, pp. 340-358, Mar. 2014.

[48] L. Fernández-Luque and T. Bau, "Health and social media: perfect storm of information.," Healthc. Inform. Res., vol. 21, no. 2, pp. 6773, Apr. 2015.

[49] E. Ferrara, O. Varol, C. Davis, F. Menczer, and A. Flammini, "The rise of social bots," Commun. ACM, vol. 59, no. 7, pp. 96-104, Jun. 2016.

[50] C. W. Schmidt, "Trending now: using social media to predict and track disease outbreaks.,"
Environ. Health Perspect., vol. 120, no. 1, pp. A30-3, Jan. 2012.

[51] X. Chen, S. C. J. Sin, Y. L. Theng, and C. S. Lee, "Why Do Social Media Users Share Misinformation?," Proc. ACM/IEEE Jt. Conf. Digit. Libr., vol. 2015-June, pp. 111-114, 2015.

[52] WWViews.org, "The World Wide Views method," 2020. [Online]. Available: http://wwviews.org/the-world-wide-viewsmethod/. [Accessed: 18-Jun-2020].

[53] J. Chae, "Virtual makeover: Selfie-taking and social media use increase selfie-editing frequency through social comparison," Comput. Human Behav., vol. 66, pp. 370-376, 2017. 